\title{
Directionality in Vowel Harmony: The Case of Karajá (Macro-Jê)
}

\author{
Eduardo Rivail Ribeiro ${ }^{1}$ \\ University of Chicago and Museu Antropológico (Universidade Federal de Goiás, \\ Brazil)
}

\section{Introduction}

This paper describes some unusual features of vowel harmony in Karajá, a Macro-Jê language from Central Brazil, outlining some of the implications they may have for a broader typological characterization of vowel harmony systems. ${ }^{2}$ Karajá presents a system of vowel harmony in terms of the feature [ATR] 'advanced tongue root'-apparently, the first documented case of [ATR] vowel harmony in a South American language (Ribeiro 2000). However, when compared with more typical cases of tongue-root harmony systems, such as the ones found in the African languages Yoruba (Archangeli \& Pulleyblank 1989) and Turkana (Noske 1995), Karajá presents some interesting peculiarities. For example, in Karajá, [ATR] alternations involve not only front and back vowels (as is typically the case with well-known ATR harmony systems), but high central

\footnotetext{
${ }^{1}$ The fieldwork on which the present paper is based was conducted under the auspices of a grant from the Tinker Foundation, administered by the Center for Latin American Studies of the University of Chicago. I would like to thank these institutions, as well as CNPq (the Brazilian National Council for Scientific Development) and the Wenner-Gren Foundation for Anthropological Research, for their financial support. My understanding of this topic has benefited greatly from discussions with Gunnar Hansson, to whom I would like to extend my acknowledgments. Any remaining shortcomings are, of course, my entire responsibility. The research reported in this paper is still in a preliminary phase, and is part of a larger project of description of the Karajá language. Any comments, suggestions, and/or criticisms would be very much appreciated.

${ }^{2}$ Karajá is spoken along the Araguaia River (an indirect tributary of the Amazon River), in the states of Goiás, Mato Grosso, Tocantins. and Pará. The phenomena described in this paper are common to all of the four Karajá dialects (Southern Karajá, Northern Karajá, Javaé, and Xambioá). The language presents systematic differences between male and female speech (Ribeiro 2001 ), but such distinctions bear no relevance for the discussion of the facts described in this paper. The data on which this paper is based are mainly from the female speech of the Southern and Northern Karajá dialects. Abbreviations: ANTI 'antipassive', CTFG 'centrifugal direction', EMPH 'emphatic', FUT 'future', IMPER 'imperative', MMPERF 'imperfective', INTR 'intransitive', LOC 'locative postposition', POT 'potential', PERF 'perfective', PROGR 'progressive', REL 'relational prefix', IRANS 'transitive'.
} 
vowels as well. ${ }^{3}$ Another interesting characteristic of Karajá vowel harmony is its extreme pervasiveness and productivity. It applies not only accross the different constituents of a compound, but across word boundaries as well.

Vowel harmony in Karajá can be roughly described as a process of regressive propagation of the feature value [+ATR] to vowels that would otherwise surface as [-ATR], such as represented schematically in (1) below. Any morpheme containing a [+ATR] vowel can trigger harmony, including stems, affixes, and clitics, a property which characterizes Karajá vowel harmony as a dominantrecessive system. However, unlike other well-known dominant-recessive vowel harmony systems, such as Turkana (Noske 1995) and Nez Perce (Rigsby \& Silverstein 1968), vowel harmony in Karajá is strictly directional, applying exclusively from right to left. Thus, in the example below, vowel harmony is triggered by the [+ATR] vowel of the imperative particle, turning preceding [-ATR] vowels into [+ATR]; notice that the [-ATR] vowel of the emphatic particle remains intact:

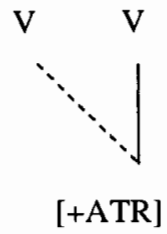

\section{$b-\varepsilon-d \varepsilon h \varepsilon=i k u d i=h \varepsilon \quad$ [bede'heikunihe] 2-INTR-look=IMPER=EMPH 'Look!'}

The fact that [-ATR] vowels can follow, but not precede [+ATR] vowels clearly shows that vowel harmony in Karajá is strictly a right-to-left process. The straightforward relevance of directionality for the description of vowel harmony in Karajá challenges theories that discard directionality as an independent parameter of assimilation, such as the one proposed by Bakovič (2000), among others (Beckman 1995, 1997, 1998; Lombardi 1996, 1999). In this view, directionality is an epiphenomenon dependent mostly on the morphological structure of the language. As I intend to show in this paper, although such an account seems to be especially appealing for stem-controlled harmony systems, as well as for more familiar examples of dominant-recessive systems, the Karajá

\footnotetext{
${ }^{3}$ In fact, some languages indeed present an [ATR] contrast between central vowels, although less commonly found than the one involving front and back vowels. That is the case of Degema (Niger-Congo), for example, which is described as having a 'complete' 10-vowel [ATR] harmony system (Fulop, Kari, \& Ladefoged 1998). In this language, the [-ATR] low vowel [a] constitutes a harmonic pair with the [+ATR] vowel [ə], in addition to the contrasts between $[1, \varepsilon, \nu, v]$ and $[\mathrm{i}, \mathrm{e}, \mathrm{o}, \mathrm{u}]$, more commonly attested. [ATR] contrast between high central vowels, such as displayed by Karajá, seems to be a rather less common phenomenon.
} 
data provide a strong counterexample to such claims, suggesting that such theories are inadequate as a universal characterization of vowel harmony phenomena.

\section{Karajá [ATR] harmony}

In previous phonological descriptions of Karajá (Fortune \& Fortune 1963; Cavalcante 1992), vowel harmony is treated as a matter of height assimilation, being briefly mentioned as a process by which a high or close-mid vowel "closes" an open-mid vowel in a preceding syllable. However, this formulation would not account for a number of cases in which a high vowel would 'fail' to trigger vowel harmony (Ribeiro 2000: 80-81). This is a direct consequence of the fact that both accounts did not consider phonological contrasts such as the ones illustrated by the minimal pairs below:

$$
\begin{array}{lll}
\text { a. } & W I & \text { 'good' } \\
\text { b. } & k \dot{ } & \text { 'bark fiber' } \\
\text { c. } & T U & \text { 'thigh; skirt' }
\end{array}
$$

$$
\begin{array}{lll}
\text { a. } & w i & \text { 'reciprocal' } \\
\text { b. } & k \dot{ } & \text { 'inside' } \\
\text { c. } & r u & \text { 'eye, sight' }
\end{array}
$$

While the high vowels in (4) behave like the close-mid vowels, triggering vowel harmony, the high vowels in (3) behave like the open-mid vowels, undergoing it. This fact demonstrates that vowel harmony in Karajá is of the 'cross-height' type, involving not height, but the [ATR] feature. According to their behavior in triggering, undergoing, or blocking vowel harmony, the vowels of Karajá can be grouped as in the table below:

(5) Vowels according to their behavior regarding vowel harmony (apud Ribeiro 2000, revised) ${ }^{4}$

\section{Oral}

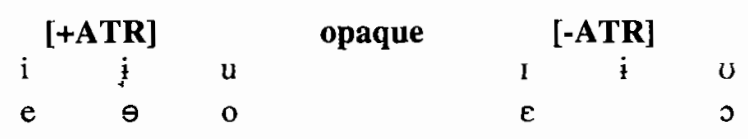

a

\footnotetext{
${ }^{4}$ One of the main phonological differences among the four dialects is that in Southern and Northern Karajá there occurs a schwa $/ \partial /$ in unstressed positions, corresponding to environments in which Xambioa and Javae present a vowel identical to the one occurring in the following syllable: Southern/Northern Karajá $d$-orədo 'tongue', bəde 'land, ground'; Javaé/Xambioá $d$-orədっ, bede. Although its occurrence in Southern and Northern Karajá is limited to unstressed syllables, the schwa can clearly be reconstructed for Proto-Karajá. Regardless of its phonemic status, however, the existence of the schwa does not alter the discussion of vowel harmony presented above, since the schwa is transparent to vowel harmony: $d$-orado=le (REL-tongue=EMPH) [dor $d o$ 'le] 'just the tongue' (Javaé/Xambioá [dorodóle]).
} 


\title{
Nasal
}

\author{
[+ATR] opaque \\ $\tilde{1} \tilde{\text { à }} \tilde{\text { o }}$ \\ $\tilde{\mathrm{a}}$
}

As shown in (5), the only oral vowels that do not have a harmonic counterpart are the low vowel $/ a /$, which is opaque (9a), and the mid-close central vowel $/ 9 /$, which is dominant (12a). As the examples in (6) below demonstrate, all combinations of vowels in a phonological word are possible, except [-ATR] vowels preceding [+ATR] vowels. When a [-ATR] vowel precedes a [+ATR] vowel, vowel harmony takes place (6d).

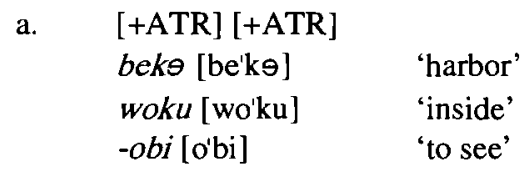

b. $\quad[+\mathrm{ATR}][-\mathrm{ATR}]$

$\begin{array}{ll}\text { bud } \varepsilon \text { [bu'd } \varepsilon] & \text { 'little, few' } \\ i \theta \varepsilon\left[\mathrm{i}^{\prime} \int \varepsilon\right] & \text { 'dance' } \\ k i \theta \varepsilon\left[\mathrm{ki} \int \varepsilon\right] & \text { 'grassy, bushy' }\end{array}$

c. [-ATR] [-ATR $]$

$\begin{array}{ll}d o r \varepsilon\left[\mathrm{d} \jmath^{\prime} \mathrm{r} \varepsilon\right] & \text { 'parrot' } \\ d \varepsilon b o[\mathrm{de} \text { 'bo] } & \text { 'hand' } \\ h \varepsilon d f \text { [he'di] } & \text { 'blanket' }\end{array}$

d. $\quad *[$-ATR $][+$ ATR $]$

$\begin{array}{llll}d o r \varepsilon & d-e \quad \text { [dore'de] } & d-\varepsilon b o \quad \text { kube } & \text { [deboku'be] } \\ \text { parrot REL-wing } & \text { REL-hand palm } & \\ \text { 'parrot's wing' } & \text { 'palm (of hand)' }\end{array}$

The process of vowel harmony in Karajá is further illustrated below by examples involving the imperfective auxiliary $=r-e$, a clitic (7), and the derivational suffix $-d \bar{t}$ 'similar to' (8). As shown in (5) above, both high and mid [-ATR] vowels undergo vowel harmony:
a. $\quad \varnothing-r-a-k s h o d \varepsilon=r-e \quad$ [rakoho'dere]
3-CTFG-INTR-hit=CTFG-IMPERF
'He/she hit.' 
Directionality in Vowel Harmony: The Case of Karajá

b. $\quad \varnothing-r-v-b \varepsilon h \varepsilon=r-e \quad$ [rube'here]

3-CTFG-INTR-go.down=CTFG-IMPERF

'He/she went down.'

c. $\varnothing$-r-a-rI=r-e [ra'rire]

3-CTFG-INTR-leave=CTFG-IMPERF

'He/she was left.'

(8)
a. brore-di [brore'ni]
deer-similar.to
b. $\quad b \varepsilon d o-d i \quad$ [bedo'ni]
'a type of filhote'
c. roku-di [raku'ni]
gourd-similar.to
'watermelon'
$\begin{array}{llr}\text { d. } & \text { hãdI-dI } & \text { [hãdi'ni] } \\ \text { jacu (fish sp.)-similar.to }\end{array}$
'a type of jacu'
e. $\quad t i-d i$
[rịni]
'cow'
cari (fish sp.)-similar.to
'a type of cari'

On the other hand, the vowels $/ \mathrm{a} /, / \tilde{\mathrm{a}} /, / \tilde{\mathrm{o}} /$, and $/ \tilde{\mathrm{a}} /$ are opaque, systematically blocking harmonization:
a. $\quad \varnothing-r-\varnothing-\varepsilon r \underline{a}=r-e \quad$ [re'rare]
3-CTFG-INTR-copulate $=$ CTFG-IMPERF
'He copulated.'
b. $\quad r-\varepsilon-h a \tilde{a} d \varepsilon=r-e$ [rehã'dere]
CTFG-1+TRANS-hit=CTFG-IMPERF
'I hit (it).'
c. $\varnothing-r-a-k \supset h \supset d \varepsilon=k \underline{o}=r-e \quad$ [rakshod $\varepsilon^{\prime}$ kõre]
3-CTFG-INTR-hit=NEG=CTFG-IMPERF
'He/she didn't hit.'
d. $r-\varepsilon-b \tilde{z}=r-e \quad$ [re'mõre]
CTFG-1+TRANS-catch/take=CTFG-IMPERF
'I caught (it).' 


\subsection{Mid versus high vowels}

As the examples above show, both high and mid [-ATR] vowels are recessive, undergoing vowel harmony. However, there is a crucial difference between both categories: while mid vowels undergo vowel harmony iteratively (7a-b, 8a-b), high vowels undergo harmony non-iteratively. That is, although high vowels do undergo vowel harmony, they fail to transmit the harmonic feature to preceding vowels:
a. $\quad k r o b r-d \tilde{\imath}$ monkey-similar.to
[krobi'ni] 'a type of monkey'
b. $\quad k o d U-d i ̄$
turtle-similar.to
[kodu'ni] 'a type of turtle'
c. hekodí-dĩ
fire-similar.to
d. koluko-di [koluko'ni] 'cedar' cajá (tree sp.)-similar.to

Also striking is the fact that the behavior of high [-ATR] vowels seems to be sensitive to morphological considerations. While in examples such as the ones above, with the suffix -di, high [-ATR] vowels apparently only undergo vowel harmony non-iteratively, examples involving clitics such as the perfective $=r e$ or the future particle $=k a r e$ can optionally harmonize iteratively:
a. $\varnothing$-r-a-hilor=kəre [rahilo'ikre] [rahịlo'ikre]
3-CTFG-INTR-vomit=FUT
'He/she will vomit.'
b. $\quad \varnothing-r-\varnothing$ - $\varepsilon b u r \varepsilon=r-e \quad$ [rebu'rere] $\sim$ [rebu'rere]
3-CTFG-INTR-get.angry=CTFG-IMPERF
'He got angry.'
c. $\quad \varnothing$-r-a-brodire=kəre [rabrodị'rekre] $\sim$ [rabrodị'rekre]
3-CTFG-INTR-bròtyre=FUT
'He/she will become bròtyre.

This 'semi-opacity' - that is, the fact that high [-ATR] vowels both undergo and block vowel harmony-may have interesting theoretical implications for output-oriented frameworks, since it prompts the need for distinguishing

\footnotetext{
${ }^{5}$ Bròtyre is a kind of ceremonial relationship (roughly similar to godparenthood).
} 


\section{Directionality in Vowel Harmony: The Case of Karajá}

underlying [high, +ATR] vowels, which trigger vowel harmony, from derived ones, which block it. This is a question to be further discussed in a future work.

\subsection{Domain}

As the data presented above suggest, vowel harmony in Karajá is extremely pervasive, applying not only in and across compounds (12), but also across word boundaries (13):

a. $\quad \varnothing-r-a-r u \varepsilon-d \theta=r-a \quad$ [rarue' $\delta \ominus r a]$

3-CTFG-INTR-eye-close $=$ CTFG-PERF

'He/she became blind.'

b. $\varnothing-r-a-w \varepsilon-b s h o=r-e \quad$ [rawebo'hore]

3-CTFG-INTR-belly-break=CTFG-IMPERF

'[They] had their bellies broken.'

$$
\begin{aligned}
& \text { a. hãlok’e Kịla [hãloko,ekị'dza] } \\
& \text { jaguar small } \\
& \text { 'small jaguar' } \\
& \text { b. wa-rikore } d-u \text { [warit]o,re'dzu] } \\
& \text { 1-offspring REL-tooth } \\
& \text { 'my child's tooth' }
\end{aligned}
$$

The domain of vowel harmony seems to be the phonological word, characterized by a single primary stress. As the example below shows, vowel harmony does not seem to apply across phonological words (14). Although stress seems to be useful in determining the domain of vowel harmony, it is irrelevant in characterizing triggers, since, as we have seen, vowel harmony can be triggered not only by stems and derivational suffixes (which are intrinsically tonic), but by clitics (which are intrinsically unstressed) as well. ${ }^{6}$

\footnotetext{
6 Vowel harmony languages commonly present disharmonic roots-mostly loanwords that 'refuse' to follow the harmonic pattern of the borrowing language. As of yet, I have not found any example of disharmonic roots in Karajá. Porential sources of disharmony seem to be systematically 'fixed up', as illustrated by the examples below. In the likely source of these loanwords (the dialects of Portuguese spoken around the Karajá territory), alveolar stops are palatalized when followed by the high front vowel [i]. As I have shown elsewhere (Ribeiro 2000: 86-88), palatal consonants in Karajá only occur in contiguity to [high, +ATR] vowels. Therefore, the loanwords below pose a conflict to Karaja phonotactic patterns, since a syllable containing an alveopalatal fricative is preceded by a [-ATR] vowel. The conflict is solved by substituting the alveolar implosive $/ d /$ for the original alveopalatal consonant:
} 


\section{Directionality}

As we have seen, the fact that [-ATR] vowels can follow, but not precede [+ATR] vowels clearly shows that vowel harmony in Karajá is strictly a right-to-left process. This is further illustrated by the examples below, involving the stems $b u d \varepsilon$ 'few, little', rikore 'offspring', duho 'to curse', and kite 'grassy'. Since these stems contain both dominant and recessive vowels, they can either trigger (a) or undergo (b) vowel harmony:
a. $\quad b a d \varepsilon-b u d \varepsilon$
$[$ bə,debu'd $\varepsilon]$
land-few
'island'
b. $\quad i-b u d \varepsilon=r-e$
[ibu'dere]
3 -few $=$ CTFG-IMPERF
'It is little.'

(16)

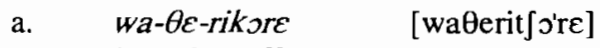
1-mother-offspring
'my sibling'
b. wa-rikore boho [warit]oreboho]
1-offspring PLURAL
'my children'

a. $\varnothing$-r-o-duho=rerI [rot]u'horer1]

3-CTFG-ANTI-curse $=$ CTFG-PROGR

'He is cursing.'

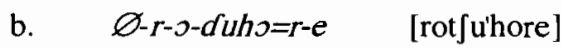

3-CTFG-ANTI-curse=CTFG-IMPERF

'He cursed.'

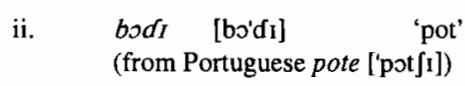


Directionality in Vowel Harmony: The Case of Karajá

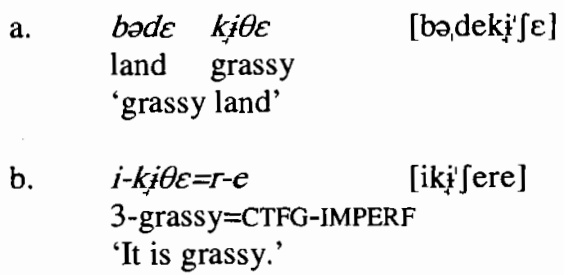

As mentioned above, examples such as these, in which directionality is clearly at play, pose an interesting challenge to theories which reject directionality as an independent parameter of assimilation, such as the one proposed by Bakovič (2000). Bakovič claims that "agreement constraints are left-right symmetrical" (p. 6), and that directionality is in fact an epiphenomenon derived from morphological considerations. This claim seems to be rather plausible in the cases of languages presenting stem-controlled vowel harmony. As he states, the majority of languages with vowel harmony (such as Turkish and Hungarian) are strictly suffixing, and present stem-controlled vowel harmony. Thus, despite the appearances that vowel harmony in these languages is unidirectional, left-to-right, this directionality would be merely a consequence of the morphological structure of the language (p. 7). In other vowel harmony languages, such as Yoruba, "morphology is strictly prefixal; the apparent right-to-left directionality of [ATR] harmony is thus a reflection of stem control" (p. 61).

As for dominant-recessive harmony systems. Bakovič's proposal seems to be based on the assumption, tacitly or explicitly stated in the literature on vowel harmony, that dominant-recessive harmony systems are inherently bidirectional. Examples such as bude 'few' and rikore 'offspring', presented above, in which directionality is clearly at play, are, according to Bakovič, 'unattested':

"If dominant-recessive harmony could in principle be unidirectional, then we would expect to find a language in which the recessive vowels on one side of a dominant vowel are affected by harmony, while those on the other side remain unaffected. Such a pattern is entirely unattested." (Bakovič 2000: 8; italics added)

The Karaja data, as we have seen, demonstrate that this is definitely not the case. Such a pattern is actually rather common in Karajá, not only in polymorphemic constructions such as (2), but in tautomorphemic words as well (15-18). Thus, Karajá provides a strong counterexample to such claims, showing that strict directionality can aiso be found in dominant-recessive vowel harmony systems, constituting in such cases an independent parameter of assimilation.

\section{Final remarks}

The discussion presented in this paper hopefully shows that Karajá, a Macro-Jê language from Brazil, presents a straightforward case of dominant-recessive vowel harmony with strict right-to-left directionality, contra the assumption that 
such systems would be always bidirectional (Bakovič 2000). Rather than describing a universal state of affairs, such an assumption probably reflects the fact that our understanding of vowel harmony systems is drawn mainly from a limited sample of languages, a number of which are genetically or geographically related. As van der Hulst and van de Weijer (1995) remind us, "our knowledge of the structure and classification of harmony systems is still extremely limited." As linguistic research expands to comprise lesser-known language areas, such as South America, a more complete picture starts to emerge, revealing otherwise 'unattested' patterns.

\section{References}

Archangeli, Diana \& Douglas Pulleyblank. 1989. Yoruba vowel harmony. Linguistic Inquiry 20.2: 173-217.

Bakovic, Eric. 2000. Harmony, dominance, and control. Doctoral dissertation. Rutgers University.

Beckman, Jill. 1995. Shona height harmony: markedness and positional identity. UMOP 18, 53-76.

Beckman, Jill. 1997. Positional faithfulness, positional neutralization, and Shona vowel harmony. Phonology 14: 1-46.

Beckman, Jill. 1998. Positional faithfulness. Doctoral dissertation, University of Massachusetts, Amherst.

Cavalcante, Marita Pôrto. 1992. Fonologia do Karajá. Revista do Museu Antropológico 1. Goiânia: Editora da UFG.

Fortune, David \& Fortune, Gretchen. 1963. The Phonemes of the Karaja Language (manuscript). Rio de Janeiro: Arquivo Linguístico do Museu Nacional.

Fulop, Sean A., E. Kari, \& P. Ladefoged. 1998. An acoustic study of the tongue root contrast in Degema vowels. Phonetica 55: 80-98.

Hulst, Harry van der \& Jeroen van der Weijer. 1995. Vowel harmony. In Goldsmith, John (ed.) Handbook of Phonological Theory, pp. 495-534. Cambridge, MA: Blackwell.

Lombardi, Linda. 1996. Restrictions on direction of voicing assimilation: an OT account. University of Maryland Working Papers in Linguistics 4: 84-102.

Lombardi, Linda. 1999. Positional faithfulness and voicing assimilation in Optimality Theory. NLLT 17, 267-302.

Noske, Manuela. 1995. The ternary use of distinctive features: In defense of the number three. PhD dissertation, Chicago: University of Chicago.

Ribeiro, Eduardo R. 2000. [ATR] vowel harmony and palatalization in Karajá. Santa Barbara Papers in Linguistics 10 (Proceedings from the $3^{\text {rd }}$ Workshop on American Indigenous Languages), pp. 80-92. Santa Barbara: UCSB. 
Directionality in Vowel Harmony: The Case of Karajá

Ribeiro, Eduardo R. 2001. Female versus male speech in Karajá. Paper presented at the meeting of the Society for the Study of the Indigenous Languages of the Americas $/ 4^{\text {th }}$ Workshop on American Indigenous Languages. Santa Barbara, July 6, 2001.

Rigsby, Bruce \& Michael Silverstein. 1969. Nez Perce vowels and ProtoSahaptian vowel harmony. Language 45.1: 45-59.

Eduardo Rivail Ribeiro

University of Chicago

Department of Linguistics

1010 E. $59^{\text {th }}$ Street

Chicago, IL 60637

erribeir@midway.uchicago.edu 